\title{
Activity of the adrenomedullin system to personalise post-discharge diuretic treatment in acute heart failure
}

\author{
Nikola Kozhuharov ${ }^{1,2,3} \cdot$ Leong $\mathrm{Ng}^{4,5}$. Desiree Wussler ${ }^{1,2,6}$. Ivo Strebel ${ }^{1,2}$. Zaid Sabti ${ }^{1,2}$. Oliver Hartmann ${ }^{7}$. \\ Mohamed Eltayeb ${ }^{4,5} \cdot$ lain Squire ${ }^{4,5} \cdot$ Albina Nowak $^{1,2,8} \cdot$ Max Rieger $^{1,2} \cdot$ Jasmin Martin $^{1,2,6}$. Eleni Michou ${ }^{1,2}$. \\ Sabrina Stefanelli, ${ }^{1,2} \cdot$ Christian Puelacher $^{1,2,6} \cdot$ Samyut Shrestha ${ }^{1,2} \cdot$ Maria Belkin $^{1,2} \cdot$ Tobias Zimmermann $^{1,2,6}$. \\ Pedro Lopez-Ayala ${ }^{1,2}$. Joachim Struck ${ }^{7}$. Andreas Bergmann ${ }^{7} \cdot$ Alexandre Mebazaa $^{9}$ - Alice Blet ${ }^{9}$. \\ Danielle Menosi Gualandro ${ }^{1,2} \cdot$ Tobias Breidthardt $^{1,2,6} \cdot$ Christian Mueller $^{1,2}$ (I)
}

Received: 21 April 2021 / Accepted: 7 July 2021 / Published online: 23 July 2021

(c) The Author(s) 2021

\begin{abstract}
Background Quantifying the activity of the adrenomedullin system might help to monitor and guide treatment in acute heart failure (AHF) patients. The aims were to (1) identify AHF patients with marked benefit or harm from specific treatments at hospital discharge and (2) predict mortality by quantifying the adrenomedullin system activity.

Methods This was a prospective multicentre study. AHF diagnosis and phenotype were centrally adjudicated by two independent cardiologists among patients presenting to the emergency department with acute dyspnoea. Adrenomedullin system activity was quantified using the biologically active component, bioactive adrenomedullin (bio-ADM), and a prohormone fragment, midregional proadrenomedullin (MR-proADM). Bio-ADM and MR-proADM concentrations were measured in a blinded fashion at presentation and at discharge. Interaction with specific treatments at discharge and the utility of these biomarkers on predicting outcomes during 365-day follow-up were assessed.

Results Among 1886 patients with adjudicated AHF, 514 patients (27.3\%) died during 365-day follow-up. After adjusting for age, creatinine, and treatment at discharge, patients with bio-ADM plasma concentrations above the median ( $>44.6$ pg/ $\mathrm{mL}$ ) derived disproportional benefit if treated with diuretics (interaction $p$ values $<0.001$ ). These findings were confirmed when quantifying adrenomedullin system activity using MR-proADM ( $n=764)$ (interaction $p$ values $<0.001)$. Patients with bio-ADM plasma concentrations above the median were at increased risk of death (hazard ratio 1.87, 95\% CI 1.57-2.24; $p<0.001)$. For predicting 365-day all-cause mortality, both biomarkers performed well, with MR-proADM presenting an even higher predictive accuracy compared to bio-ADM $(p<0.001)$.

Conclusions Quantifying the adrenomedullin's system activity may help to personalise post-discharge diuretic treatment and enable accurate risk-prediction in AHF.
\end{abstract}

Keywords Dyspnoea $\cdot$ Acute heart failure $\cdot$ Adrenomedullin $\cdot$ Diuretics

\section{Introduction}

Acute heart failure (AHF) is the most common cause of hospitalisation in patients 50 years or older, and it is still associated with unacceptably high mortality and morbidity [1, 2],

Nikola Kozhuharov and Leong Ng have contributed equally and should be considered the first authors.

Christian Mueller

christian.mueller@usb.ch

Extended author information available on the last page of the article with up to $30 \%$ of patients dying within 1 year after hospital discharge [1]. The poor outcomes of patients with AHF are at least in part due to incomplete understanding of the pathophysiology of AHF, as well as uncertainty in defining the intensity of both in-hospital and immediate post-discharge management resulting from a lack of high-quality evidence for treatments in these clinical settings $[1,3]$.

The use of cardiovascular biomarkers may contribute to addressing some of these unmet clinical needs. For instance, the clinical introduction of natriuretic peptides quantifying haemodynamic cardiac stress has substantially improved early and accurate diagnosis of AHF [4-7]. Novel 
cardiovascular biomarkers may help to unravel distinct pathophysiological AHF phenotypes with marked benefits (or harm) from specific treatments and allow personalised treatment and management. Initial pilot studies suggested that the adrenomedullin system may allow quantifying systemic microvascular dysfunction and the associated mortality risk [8-13]. Specifically, adrenomedullin secretion is increased upon volume overload and is expected to function as a compensatory mechanism maintaining barrier function, thus hindering tissue congestion [14]. In this context, a clinical phase-2 trial with a monoclonal antibody against the $\mathrm{N}$-terminal end of adrenomedullin (adrecizumab), which increases the plasma concentrations of bioactive adrenomedullin, has recently been initiated in AHF patients (NCT04252937) [15]. Furthermore, since residual congestion in AHF is associated with adverse outcomes, and evidence from large clinical trials guiding the use of diuretics is generally lacking, the ability of adrenomedullin to reflect tissue congestion may be particularly useful for this purpose [16].

In an international multicentre study, we aimed to use two complimentary biochemical windows to quantify the activity of the adrenomedullin system to: (1) identify AHF patients with marked benefit or harm from specific treatments at hospital discharge, and (2) predict mortality. The first biomarker used was midregional proadrenomedullin (MR-proADM), a stable precursor that does not distinguish between biologically active amidated adrenomedullin and the non-functional adrenomedullin variant containing a glycine-extended C-terminal residue [15]. The second biomarker was the biologically active form of adrenomedullin, bioactive adrenomedullin (bio-ADM) [17].

\section{Methods}

\section{Study design and adjudication of final diagnosis}

The study sample included three prospective diagnostic cohorts of unselected AHF patients admitted after presenting with acute dyspnoea to the emergency department (ED) of the respective participating university hospitals in three countries using comparable methodology (United Kingdom, France, and Switzerland NCT01831115). Assessment and therapies for AHF, including diuretics, were provided according to guidelines and the discretion of attending physicians [6]. AHF was centrally adjudicated by two independent cardiologists in accordance with European Society of Cardiology guidelines [6]. Inclusion was independent of renal function, although patients with terminal renal failure on renal replacement therapy were excluded. Patients were followed for at least 1 year. This study was performed according to the principles of the Declaration of Helsinki and ethics approval was granted from the respective research ethics committees. All patients provided written informed consent. The authors designed the study, gathered, and analysed the data according to The TRIPOD Statement for studies reporting multivariable prediction models for individual prognosis (Supplemental Table 1), vouch for the data and analysis, wrote the paper, and decided to publish.

\section{Plasma sampling}

After obtaining signed informed consent, venous blood was drawn from recumbent patients and collected in tubes containing ethylenediaminetetraacetic acid as anticoagulant. The interval for sample obtainment at admission was up to $4 \mathrm{~h}$ (Paris), $2 \mathrm{~h}$ (Basel), and $12 \mathrm{~h}$ (Leicester) after ED presentation. Bio-ADM and MR-proADM blood samples at hospital (acute ward) discharge were available for patients recruited in Basel and Leicester. Furthermore, due to funding as well as logistics reasons, MR-proADM measurements were available only in a subgroup of unselected patients. To maximise the generalisability of the findings and considering substantial variation in overall length of hospital stay and the availability of rehabilitation units among different countries, "hospital discharge" samples were obtained at discharge from the acute ward, prior to possible transfer to a rehabilitation unit. Plasma was stored at $-80^{\circ} \mathrm{C}$ until blinded analysis in a central laboratory.

\section{Imaging, renal function, haemoconcentration, and biomarker assays}

Transthoracic echocardiography was performed using standard techniques and the left ventricular ejection fraction (LVEF) was calculated using the biplane method of discs formula. According to the LVEF, patients were stratified as heart failure with preserved ejection fraction (HFpEF, LVEF $\geq 50 \%$ ), mid-range ejection fraction (HFmrEF, LVEF 40-49), and reduced ejection fraction (HFrEF, LVEF $<40 \%$ ). Imaging data were available for patients recruited in Leicester and Basel. Haemoconcentration was defined as an increase in at least three of the four haemoconcentration-defining parameters (haemoglobin, haematocrit, albumin, and total protein) above admission values occurring simultaneously at discharge, as described previously [18]. These parameters as well as clinical data on volume overload such as estimated jugular venous pressure, periphery oedema, or third heart sound were only available in patients recruited in Basel. To determine plasma bio-ADM concentrations, a new double-monoclonal antibody sandwich immunoassay was used (sphingotec $\mathrm{GmbH}$, Hennigsdorf, Germany). This immunoassay selectively detects the C-terminally amidated form of adrenomedullin. In healthy subjects, the median value was previously determined to be 
$24.7 \mathrm{pg} / \mathrm{mL}$ and the 99 th percentile $43 \mathrm{pg} / \mathrm{mL}$. The lower detection limit is $3 \mathrm{pg} / \mathrm{mL}$, and intra- and interassay coefficients were 5-10\%, and 4-8\%, respectively, in the above normal measuring range. The analytical assay sensitivity is $2 \mathrm{ng} / \mathrm{L}[12,19]$. MR-proADM was measured using an automated sandwich chemiluminescence immunoassay on the KRYPTOR system (B·R·A·H·M·S AG, Hennigsdorf/Berlin, Germany), with a quantification limit of $0.23 \mathrm{nmol} / \mathrm{L}$, a within-run imprecision (coefficient of variation) of $1.9 \%$, and a between-run imprecision (coefficient of variation) of $9.8 \%[9,20]$.

\section{Outcome measures}

The two co-primary objectives were: (1) identification of AHF phenotypes with disproportional benefit or harm from medical treatment at discharge in terms of all-cause mortality during the 365-day follow-up. This was assessed by exploring interactions between bio-ADM plasma concentrations and treatment with diuretics, angiotensin-convertingenzyme (ACE) inhibitors or angiotensin receptor blockers (ARBs), beta blockers, and aldosterone-antagonists at discharge. (2) Assessment of the prognostic accuracy of bioADM for predicting all-cause mortality during the 365-day follow-up. The secondary objectives were defined as (1) and (2) with the combined outcome measure of all-cause mortality and AHF readmissions during follow-up. An additional aim was to directly compare bio-ADM with MRproADM in these indications. Furthermore, the incremental value of both biomarkers was assessed when added to the OPTIMIZE-HF clinical risk-score, established for predicting up to 90-day all-cause mortality after hospitalisation for AHF [21]. Similarly, the independent predictive value of bio-ADM and MR-proADM was compared to the impact of haemoconcentration during hospitalisation [18].

Endpoints were ascertained blinded to biomarker data from hospital records and electronic databases. Patients who survived until discharge were followed for at least 365 days after the initial hospitalisation. Data on AHF readmissions were not available for patients from Paris $(n=225,11 \%$ of the overall cohort).

\section{Statistical analysis}

The Kolmogorov-Smirnov test and visual inspection of the distribution of variables was used for testing normality. Continuous variables are presented as medians with interquartile range, and categorical variables as numbers and percentages. Comparisons between groups were made using Chi-square, Mann-Whitney $U$, and Kruskal-Wallis tests, as appropriate. Spearman's rho was used to analyse correlations. One-way ANOVA and Eta test statistics were used to assess associations of categorical variables and continuous variables. All-cause mortality and its combination with AHF hospitalisations during follow-up were plotted in Kaplan-Meier curves, and the log-rank test was used to assess differences between groups. The interaction $p$ values between biomarker plasma concentrations and the predefined subgroups according to medication at discharge were calculated in multivariable models using Cox proportional hazards analysis. Further adjustment of these multivariable models was imposed for clinical considerations: the variables age and creatinine plasma concentrations at discharge were incorporated, as both may affect the prescription of heart failure drugs and mortality. Severe renal dysfunction and hypotension (systolic blood pressure below $90 \mathrm{mmHg}$ ) were considered clinical criteria, possibly justifying temporary withdrawal of diuretic therapy. In a subgroup of patients with available haemoconcentration data, this parameter as well as bio-ADM or MR-proADM and variables from a validated risk model to predict 365-day all-cause mortality were entered in multivariable regression models [22]. Sensitivity analysis was performed after excluding patients discharged from acute wards to palliative care. Hazard ratios (HR) are presented with $95 \%$ confidence intervals (CI). The prognostic accuracy of bio-ADM and MR-proADM plasma concentrations were quantified using the area under the timedependent receiver-operating characteristic curves (AUC) and compared as described previously [23]. Furthermore, time-dependent receiver-operating characteristic curves were also used to assess the prognostic accuracy of the OPTIMIZE-HF risk-score alone and its combination with each biomarker. This was a post hoc analysis within prospective studies, and the sample size of the overall cohort was not determined specifically for this analysis [18]. No imputation was performed for missing values. Patients without complete clinical follow-up were censored at the time of the last known contact. All hypothesis testing was 2-sided and after Bonferroni correction for multiple testing (16 tests for interactions for the primary endpoint of all-cause mortality) a $p$ value $\leq 0.003$ was considered significant. Statistical analyses were performed using SPSS version 25 , R version 3.5.1 ("timeROC").

\section{Results}

\section{Patient characteristics and biomarkers}

A total of 1886 AHF patients enrolled between March 2006 and June 2015 were eligible for this analysis (Supplemental Fig. 1); $37.9 \%$ were women and the median age was 78 years (Table 1); $34.8 \%$ patients had HFpEF, $14.2 \%$ had HFmrEF, and 20.1\% had HFrEF. Median bioADM concentrations at admission were $44.6 \mathrm{pg} / \mathrm{mL}$, being similar between women and men $(p=0.219)$. Bio-ADM 
Table 1 Patient characteristics according to study site

\begin{tabular}{|c|c|c|c|c|}
\hline & All patients $(n=1886)$ & Leicester $(n=862)$ & Paris $(n=215)$ & Basel $(n=809)$ \\
\hline \multicolumn{5}{|l|}{ Demographics } \\
\hline Age, years & $78.0(69.0-84.0)$ & $77.0(68.1-83.0)$ & $76.0(66.0-84.0)$ & $79.0(70.0-85.0)$ \\
\hline Female, gender, $\%$ & 37.9 & 37.4 & 38.6 & 38.2 \\
\hline BMI, $\mathrm{kg} / \mathrm{m}^{2}$ & $27.4(24.1-31.6)$ & $31.4(27.2-37.7)$ & $*$ & $26.5(23.5-30.1)$ \\
\hline \multicolumn{5}{|l|}{ Clinical parameters at ED } \\
\hline $\mathrm{SBP}, \mathrm{mmHg}$ & $134(117-153)$ & $134(116-151)$ & $135.0(113-158)$ & $135(118-154)$ \\
\hline $\mathrm{HR}$, beats/min & $87(72-105)$ & $88(73-105)$ & $85(69-105)$ & $85(71-103)$ \\
\hline LVEF, \% & $39(27-52)$ & $35(25-47)$ & $*$ & $44(30-55)$ \\
\hline LVEDD, cm & $5.3(4.6-5.9)$ & $5.3(4.7-5.9)$ & $*$ & $5.2(4.6-5.9)$ \\
\hline LVESD, cm & $4.2(3.3-5.1)$ & $4.4(3.6-5.2)$ & $*$ & $3.8(3.0-4.9)$ \\
\hline \multicolumn{5}{|l|}{ Medical history } \\
\hline $\mathrm{CKD}, \%$ & 33.1 & 21.7 & 20.9 & 48.6 \\
\hline Hypertension, $\%$ & 69.7 & 58.6 & 64.2 & 82.9 \\
\hline Dyslipidaemia, \% & 42.0 & 26.2 & $*$ & 59.5 \\
\hline Stroke or TIA, $\%$ & 16.2 & 16.9 & $*$ & 15.3 \\
\hline Current or ex-smoker, $\%$ & 55.4 & 48.0 & $*$ & 63.7 \\
\hline Atrial fibrillation, $\%$ & 42.7 & 47.3 & $*$ & 37.8 \\
\hline PAD, $\%$ & 10.6 & 5.3 & $*$ & 16.4 \\
\hline COPD, $\%$ & 16.1 & 10.2 & $*$ & 22.5 \\
\hline Diabetes, $\%$ & 32.0 & 34.6 & 29.3 & 30.0 \\
\hline \multicolumn{5}{|l|}{ Medication at presentation } \\
\hline ACE inhibitors/ARB, $\%$ & 61.5 & 57.0 & $*$ & 66.3 \\
\hline Beta blockers, $\%$ & 53.6 & 44.2 & $*$ & 63.8 \\
\hline Aldosterone antagonists, $\%$ & 13.1 & 12.5 & $*$ & 13.7 \\
\hline Loop diuretics, $\%$ & 65.4 & 61.1 & $*$ & 70.0 \\
\hline \multicolumn{5}{|l|}{ Medication at discharge } \\
\hline ACE inhibitors/ARB, $\%$ & 69.6 & 63.9 & 63.7 & 78.1 \\
\hline Beta blockers, $\%$ & 62.3 & 53.2 & 61.4 & 72.4 \\
\hline Aldosterone antagonists, $\%$ & 31.0 & 32.9 & 26.0 & 30.3 \\
\hline Loop diuretics, \% & 85.0 & 81.3 & 78.1 & 91.1 \\
\hline \multicolumn{5}{|c|}{ Laboratory parameters at admission } \\
\hline Haemoglobin, g/L & $125.0(111.0-138.0)$ & $123.0(109.0-137.0)$ & $*$ & $126.0(113.0-139.0)$ \\
\hline Sodium, $\mathrm{mmol} / \mathrm{L}$ & $139.0(136.0-141.0)$ & $138.0(135.0-141.0)$ & $137.0(134.0-140.0)$ & $139.0(137.0-142.0)$ \\
\hline Potassium, mmol/L & $4.3(3.9-4.6)$ & $4.4(4.0-4.7)$ & $*$ & $4.2(3.8-4.5)$ \\
\hline Creatinine, $\mu \mathrm{mol} / \mathrm{L}$ & $111.0(86.0-146.0)$ & $113.0(91.0-142.0)$ & $115.0(84.3-151.0)$ & $107.0(82.0-148.0)$ \\
\hline NT-proBNP, pg/mL & $3094(1468-6257)$ & $2188(984-4093)$ & $*$ & 4914 (2411-9787) \\
\hline $\mathrm{BNP}, \mathrm{pg} / \mathrm{mL}$ & $1244(638-2376)$ & $*$ & $1244(638-2376)$ & $*$ \\
\hline Urea, $\mathrm{mmol} / \mathrm{L}$ & $9.2(6.6-13.4)$ & $8.9(6.5-12.9)$ & $9.4(6.8-14.6)$ & $9.6(6.6-13.4)$ \\
\hline Bio-ADM, pg/mL & $44.6(30.2-69.2)$ & $49.6(33.8-81.2)$ & $47.4(33.8-82.8)$ & $38.5(26.3-57.4)$ \\
\hline MR-proADM, nmol/L & $1.67(1.20-2.42)$ & $1.74(1.30-2.69)$ & $1.18(1.04-1.78)$ & $1.62(1.16-2.23)$ \\
\hline \multicolumn{5}{|c|}{ Laboratory parameters at discharge } \\
\hline Creatinine, $\mu \mathrm{mol} / \mathrm{L}$ & $111.0(88.0-147.0)$ & $113.0(92.0-146.5)$ & $*$ & $108.0(84.0-149.0)$ \\
\hline NT-proBNP, pg/mL & $1963(861-4335)$ & $1544(644-2888)$ & $*$ & $2636(1069-5844)$ \\
\hline Bio-ADM, pg/mL & $37.0(25.8-55.6)$ & $45.6(31.6-68.9)$ & $*$ & $29.3(20.0-41.6)$ \\
\hline MR-proADM, nmol/L & $1.42(1.03-2.00)$ & $1.67(1.16-2.24)$ & $*$ & $1.28(0.96-1.78)$ \\
\hline
\end{tabular}

$A C E$ angiotensin-converting-enzyme, $A R B s$ angiotensin receptor blocker, $B M I$ body mass index, bio- $A D M$ bioactive adrenomedullin, $C A D$ coronary artery disease, $C C B$ calcium channel blockers, $C O P D$ chronic obstructive pulmonary disease, $C K D$ chronic kidney disease, $E D$ emergency department, $H R$ heart rate, $M R$-proADM midregional proadrenomedullin, $L V$ left ventricle, $L V E D D$ left ventricular end diastolic diameter, $N T$ proBNP N-terminal pro-B-type natriuretic peptide, $P A D$ peripheral artery disease, $S B P$ systolic blood pressure

*Data not available 
and MR-proADM concentrations correlated moderately $(r=0.639)$, and bio-ADM correlated less strongly with age, NT-proBNP, and serum creatinine versus MRproADM concentrations (Table 2).

\section{Survival analysis}

During the 365-day follow-up, there were 514 deaths (27.3\%). Mortality was comparable among sites (Supplemental Figs. 2 and 3). Patients who died during follow-up were older, had a lower body mass index, more often had a history of renal impairment, and lower initial systolic blood pressure and LVEF (Table 3). Notably, their bioADM and MR-proADM plasma concentrations at admission and at hospital discharge were substantially higher compared to survivors.

\section{Interaction with heart failure treatment at discharge}

There were statistically significant interactions regarding mortality depending on MR-proADM or bio-ADM plasma concentrations at admission or discharge and prescription of diuretics at discharge (Supplemental Table 2). These interactions remained significant after adjusting for age, NT-proBNP and creatinine plasma concentrations at discharge (Table 4). Overall, 276 (15\%) patients were discharged without prescription of diuretics (Supplemental Table 3). Among them, history of chronic kidney disease was less common as compared to those with diuretics prescription at discharge $(26.5 \%$ vs $33.9 \%, p=0.017)$. Similarly, treatment with diuretics prior to admission was less common $(53.1 \%$ vs $67.2 \%, p<0.001)$. Patients not receiving diuretics at discharge and with bio-ADM levels above the median presented much higher all-cause mortality (Fig. 1). Findings regarding 365-day all-cause mortality and AHF rehospitalisations were comparable (Supplemental Tables 4 and 5; Supplemental Figs. 4 and 5). Furthermore, subgroup analyses according to LVEF groups suggested more pronounced interactions in patients with HFrEF (Supplemental Tables 6 and 7).

Notably, no statistically significant interactions were present between NT-proBNP plasma concentrations and medical therapies at discharge (Supplemental Tables 8 and 9). In-depth review of clinical characteristics of patients with bio-ADM concentrations above the median and without diuretics at discharge, who died during follow-up, showed that at acute ward discharge many did not have an established medical cause such as severe renal dysfunction or hypotension, possibly justifying withdrawal of diuretics (Supplemental Table 10).
Table 2 (A) Spearman's rank correlation analysis between bio-ADM and MR-proADM and demographics, clinical characteristics, echocardiographic parameters, and biomarkers; (B) Eta test statistics to assess association between bio-ADM and MR-proADM and clinical characteristics

\begin{tabular}{|c|c|c|c|}
\hline (A) & Bio-ADM & MR-proADM & NT-proBNP \\
\hline \multicolumn{4}{|l|}{ Age, years } \\
\hline Spearman's rank (rs) & -0.058 & 0.264 & 0.217 \\
\hline$p$ value & 0.012 & $<0.001$ & $<0.001$ \\
\hline$n$ & 1885 & 764 & 1576 \\
\hline \multicolumn{4}{|l|}{ BMI, $\mathrm{kg} / \mathrm{m}^{2}$} \\
\hline Spearman's rank (rs) & 0.295 & -0.021 & -0.385 \\
\hline$p$ value & $<0.001$ & .596 & $<0.001$ \\
\hline$n$ & 1005 & 621 & 923 \\
\hline \multicolumn{4}{|l|}{ Pulse oximetry, $\%$} \\
\hline Spearman's rank (rs) & -0.075 & -0.044 & -0.081 \\
\hline$p$ value & 0.036 & 0.373 & .031 \\
\hline$n$ & 786 & 416 & 710 \\
\hline \multicolumn{4}{|l|}{$\mathrm{HR}, \mathrm{bpm}$} \\
\hline Spearman's rank (rs) & 0.001 & -0.068 & -0.012 \\
\hline$p$ value & 0.981 & 0.063 & 0.643 \\
\hline$n$ & 1814 & 755 & 1522 \\
\hline \multicolumn{4}{|l|}{$\mathrm{SBP}, \mathrm{mmHg}$} \\
\hline Spearman's rank (rs) & -0.184 & -0.248 & -0.137 \\
\hline$p$ value & $<0.001$ & $<0.001$ & $<0.001$ \\
\hline$n$ & 1815 & 750 & 1519 \\
\hline \multicolumn{4}{|l|}{$\mathrm{DBP}, \mathrm{mmHg}$} \\
\hline Spearman's rank (rs) & -0.109 & -0.188 & -0.008 \\
\hline$p$ value & $<0.001$ & $<0.001$ & 0.769 \\
\hline$n$ & 1811 & 750 & 1516 \\
\hline \multicolumn{4}{|l|}{ LV ejection fraction, $\%$} \\
\hline Spearman’s rank (rs) & -0.123 & -0.127 & -0.222 \\
\hline$p$ value & $<0.001$ & 0.003 & $<0.001$ \\
\hline$n$ & 1302 & 542 & 1234 \\
\hline \multicolumn{4}{|l|}{ LVEDD, mm* } \\
\hline Spearman’s rank (rs) & 0.057 & 0.046 & 0.144 \\
\hline$p$ value & 0.049 & 0.304 & $<0.001$ \\
\hline$n$ & 1209 & 505 & 1153 \\
\hline \multicolumn{4}{|l|}{ Haemoglobin, g/L } \\
\hline Spearman’s rank (rs) & -0.131 & -0.272 & -0.111 \\
\hline$p$ value & $<0.001$ & $<0.001$ & $<0.001$ \\
\hline$n$ & 1567 & 728 & 1496 \\
\hline \multicolumn{4}{|l|}{ Sodium, mmol/L } \\
\hline Spearman’s rank (rs) & -0.133 & -0.096 & 0.015 \\
\hline$p$ value & $<0.001$ & .008 & 0.542 \\
\hline$n$ & 1855 & 747 & 1557 \\
\hline \multicolumn{4}{|l|}{ Creatinine, $\mu \mathrm{mol} / \mathrm{L}$} \\
\hline Spearman’s rank (rs) & 0.365 & 0.639 & 0.327 \\
\hline$p$ value & $<0.001$ & $<0.001$ & $<0.001$ \\
\hline$n$ & 1855 & 749 & 1561 \\
\hline \multicolumn{4}{|l|}{ NT-proBNP, pg/mL } \\
\hline Spearman's rank (rs) & 0.089 & 0.477 & - \\
\hline
\end{tabular}


Table 2 (continued)

\begin{tabular}{llll}
\hline (A) & Bio-ADM & MR-proADM & NT-proBNP \\
\hline$p$ value & $<0.001$ & $<0.001$ & \\
\hline$n$ & 1576 & 699 & \\
MR-proADM, nmol/L & & & \\
Spearman's rank (rs) & 0.639 & - & \\
$p$ value & $<0.001$ & & \\
$n$ & 764 & & \\
(B) & & & $<0.001$ \\
Elevated JVP* & & & 0.887 \\
Eta squared $\left(\eta^{2}\right)$ & 0.011 & 0.047 & 651 \\
$\quad p$ value & 0.004 & $<0.001$ & \\
$\quad n$ & 728 & 396 & $<0.001$ \\
Third heart sound (S3)* & & & 0.822 \\
Eta squared $\left(\eta^{2}\right)$ & 0.003 & 0.005 & 681 \\
$\quad p$ value & 0.151 & 0.151 & \\
$n$ & 756 & 406 & 0.001 \\
Peripheral oedema* & & & 0.473 \\
Eta squared $\left(\eta^{2}\right)$ & 0.053 & 0.055 & 711 \\
$p$ value & $<0.001$ & $<0.001$ & \\
$n$ & 792 & 421 & \\
\hline
\end{tabular}

Correlation was calculated with measurements obtained on admission bio- $A D M$ bioactive adrenomedullin, $B M I$ body mass index, $D B P$ diastolic blood pressure, $L V$ left ventricle, $L V E D D$ left ventricular end diastolic diameter, $M R$-proADM midregional proadrenomedullin, $S B P$ systolic blood pressure, JVP jugular venous pressure, $M R$ proADM midregional proadrenomedullin

*Data available only for patients from Basel

\section{Prognostic accuracy of bio-ADM and MR-proADM}

In 764 patients (41\%), both MR-proADM and bio-ADM were measured. The prognostic accuracy for predicting allcause mortality and the combination of all-cause mortality or AHF rehospitalisations at 365 days, quantified by the AUC, was significantly higher for admission MR-proADM compared to bio-ADM (Fig. 2 and Supplemental Fig. 6; for both outcome measures: $p<0.001$ at 90,180 , and 365 days). Patients with bio-ADM concentrations above the median at admission were at increased risk of 365-day all-cause mortality (HR 1.87, 95\% CI 1.57-2.24; $p<0.001$ ). In the subgroup analysis of patients with MR-proADM measurements at admission, plasma concentrations of this biomarker above the median $(>1.67 \mathrm{nmol} / \mathrm{L})$ were associated with a higher 365-day all-cause mortality (HR 3.88, 95\% CI: 2.77-5.44; $p<0.001$; Supplemental Fig. 7). The OPTIMIZE-HF riskscore was calculated in 1706 patients (90\%), showing significant improvement in prognostic accuracy when combined with bio-ADM or MR-proADM along 90 days of follow-up (Supplemental Figs. 8 and 9). Variables from a validated risk model to predict all-cause mortality at 365 days as well as haemoconcentration achieved during hospitalisation and
bio-ADM or MR-proADM were entered in multivariable regression models (Supplemental Tables 10A and 10B). Notably, both bio-ADM and MR-proADM remained independent predictors of 365-day all-cause mortality.

\section{Sensitivity analysis}

Sensitivity analysis excluding the subgroup of patients discharged from the acute ward to palliative care $(n=10)$ revealed similar findings as in the overall cohort (Supplemental Tables 11-17 and Supplemental Figs. 10-16).

\section{Discussion}

In this large international multicentre study, we used plasma concentrations of bio-ADM and MR-proADM to identify AHF phenotypes with disproportional benefit or harm from specific heart failure treatments at hospital discharge and to predict mortality. We report five major findings.

First, patients with bio-ADM concentrations above the median had much higher mortality if not treated with diuretics at discharge. Second, medical reasons including severe renal dysfunction and/or hypotension which could possibly justify temporal withdrawal of diuretics were absent in most of these patients. This confirms the possible clinical utility of this marker to avoid medical errors such as the withdrawal of diuretics in patients who very likely derive benefits from these drugs. Third, increased bio-ADM concentrations were associated with increased risk of death at 365 days. Notably, bio-ADM remained an independent prognostic factor for all-cause mortality after adjusting for haemoconcentration, comorbidities, vital signs, and further biomarkers from a validated multivariable prediction model [22]. Fourth, comparison of the two biochemical methods assessing adrenomedullin system activity showed that MR-proADM was superior to bio-ADM in the prediction of death and/or AHF re-hospitalisation within 365 days. Fifth, both bio-ADM and MR-proADM improved the prognostic accuracy of the OPTIMIZE-HF clinical risk-score for predicting all-cause mortality within 90 days after AHF hospitalisation.

These findings extend and corroborate earlier studies investigating the importance of adrenomedullin in patients with acute dyspnoea, as well as efforts to personalise treatment in AHF patients [9, 12, 13, 17, 24-27]. The observation that patients with high bio-ADM concentrations had much higher mortality rates if not treated with diuretics at discharge seems to have immediate clinical consequences. In-depth review of the clinical characteristics of these patients indicates inappropriate clinical reaction to temporary worsening of renal function during AHF treatment, as well as a lack of reassessment of eligibility for diuretic therapy after appropriate temporal withdrawal 
Table 3 Patient's characteristics according to survival status at 365 days
Dead at 365 days $(n=514)$

Alive at 365 days $(n=1372)$

$p$ value

Demographics

Age, years

$81.0(75.0-87.0)$

Female, gender, \%

37.0

BMI, $\mathrm{kg} / \mathrm{m}^{2}$

$24.9(21.8-28.4)$

$6.5(67.0-83.0)$

$<0.001$

Clinical parameters at ED

$\mathrm{SBP}, \mathrm{mmHg}$

$126(110-145)$

85 (72-101)

$\mathrm{HR}$, beats/min

35 (24-49)

$5.3(4.6-6.1)$

LVEDD, cm

$4.2(3.4-5.2)$

LVESD, cm

Medical history

CKD, \%

44.3

Hypertension, \%

67.7

Dyslipidaemia, \%

40.7

Stroke or TIA, \%

21.3

Current or ex-smoker, \% $\quad 57.5$

PAD, \%

13.0

Atrial fibrillation, $\%$

41.4

COPD, $\%$

17.9

Diabetes, \%

31.7

38.2

0.684

$28.1(24.6-32.8)$

$<0.001$

137 (120-155)

$<0.001$

87 (72-105)

0.159

$40(29-54)$

$<0.001$

$5.2(4.6-5.9)$

0.313

$4.2(3.3-5.0)$

0.326

Medication at presentation

ACE inhibitors or ARB, \%

59.2

28.9

$<0.001$

70.4

0.280

42.4

0.560

14.3

$<0.001$

54.6

0.330

9.7

0.065

43.1

0.568

15.5

0.260

32.1

0.911

Beta blockers, $\%$

62.3

0.284

Aldosterone antagonists, $\%$

52.1

54.1

0.511

Loop diuretics, \%

16.9

11.7

0.006

Medication at discharge

ACE inhibitors or ARB, \% 51.6

61.0

$<0.001$

Beta blockers, \%

76.3

$<0.001$

Aldosterone antagonists, $\% \quad 25.7$

68.3

$<0.001$

Loop diuretics, $\%$

75.8

33.0

0.003

88.3

$<0.001$

Laboratory parameters at admission

$\begin{array}{ll}\text { Haemoglobin, g/L } & 120(108-132) \\ \text { Sodium, mmol/L } & 138(134-141) \\ \text { Potassium, mmol/L } & 4.4(3.9-4.8) \\ \text { Creatinine, } \mu \mathrm{mol} / \mathrm{L} & 132(100.0-179.0) \\ \text { Urea, mmol/L } & 12.3(8.7-17.7) \\ \text { NT-proBNP, pg/mL } & 4723.0(2360-9029) \\ \text { BNP, pg/mL } & 1897(810-3036) \\ \text { Bio-ADM, pg/mL } & 57.2(35.3-94.3) \\ \text { MR-proADM, nmol/L } & 2.32(1.68-3.07) \\ \text { Laboratory parameters at discharge } \\ \text { Creatinine, } \mu \mathrm{mol} / \mathrm{L} & 132.0(100.0-191.0) \\ \text { NT-proBNP, pg/mL } & 3327(1678-8348) \\ \text { Bio-ADM, pg/mL } & 46.7(29.0-72.5) \\ \text { MR-proADM, nmol/L } & 1.79(1.45-2.77)\end{array}$

$127(113-139)$
$139(136-141)$
$4.2(3.9-4.6)$
$105.0(83.0-134.0)$
$8.4(6.2-11.7)$
$2629.0(1259.0-5346)$
$1120(605-2128)$
$41.5(28.8-62.0)$
$1.50(1.14-2.14)$

$<0.001$

$<0.001$ 0.006

$<0.001$

$<0.001$

$<0.001$ 0.009

$<0.001$

$<0.001$

$107.0(85.0-135.0)$

$<0.001$

1659 (686-3417)

$<0.001$

$34.74(25.2-51.7)$

$<0.001$

$1.29(0.99-1.83)$

$A C E$ angiotensin-converting-enzyme, $A R B s$ angiotensin receptor blocker, $B M I$ body mass index, bio- $A D M$ bioactive adrenomedullin, $C A D$ coronary artery disease, $C C B$ calcium channel blockers, $C O P D$ chronic obstructive pulmonary disease, $C K D$ chronic kidney disease, $E D$ emergency department, $H R$ heart rate, $M R$-proADM midregional proadrenomedullin, $L V E F$ left ventricular ejection fraction, $L V E D D$ left ventricular end diastolic diameter, NT-proBNP N-terminal pro-B-type natriuretic peptide, $P A D$ peripheral artery disease, $S B P$ systolic blood pressure 
Table 4 Interaction $p$ values in multivariable models using Cox proportional hazard analysis for predicting 365-day all-cause mortality including age, bio-ADM or MR-proADM, NT-proBNP at discharge, creatinine at discharge, and medication at discharge

\begin{tabular}{lcccc}
\hline & Diuretics & ACE inhibitors or ARB & $\begin{array}{c}\text { Beta blockers } \\
\text { Aldosterone } \\
\text { antagonists }\end{array}$ \\
\hline lg bio-ADM at admission, $\mathrm{pg} / \mathrm{mL}$ & $\mathbf{< 0 . 0 0 1}$ & 0.104 & 0.541 & 0.512 \\
$\lg$ bio-ADM at discharge, $\mathrm{pg} / \mathrm{mL}$ & $\mathbf{0 . 0 0 1}$ & 0.008 & 0.609 & 0.354 \\
$\lg$ MR-proADM at admission, $\mathrm{nmol} / \mathrm{L}$ & 0.112 & 0.404 & 0.724 & 0.141 \\
$\lg$ MR-proADM at discharge, $\mathrm{nmol} / \mathrm{L}$ & $\mathbf{0 . 0 0 2}$ & 0.225 & 0.969 & 0.381 \\
\hline
\end{tabular}

$A C E$ angiotensin-converting-enzyme, $A R B s$ angiotensin receptor blocker, bio- $A D M$ bioactive adrenomedullin, $M R$-proADM midregional proadrenomedullin, NT-proBNP N-terminal pro-B-type natriuretic peptide

The bold $\mathrm{p}$ values are the ones considered significant, as reported in the Methods section: All hypothesis testing was 2-sidedand after Bonferroni correction for multiple testing (16 tests for interactions for the primary endpoint of all-cause mortality) a pvalue $\leq 0.003$ was considered significant. Please include the footnote: The bold $\mathrm{p}$ values $(\leq 0.003)$ are considered significant.
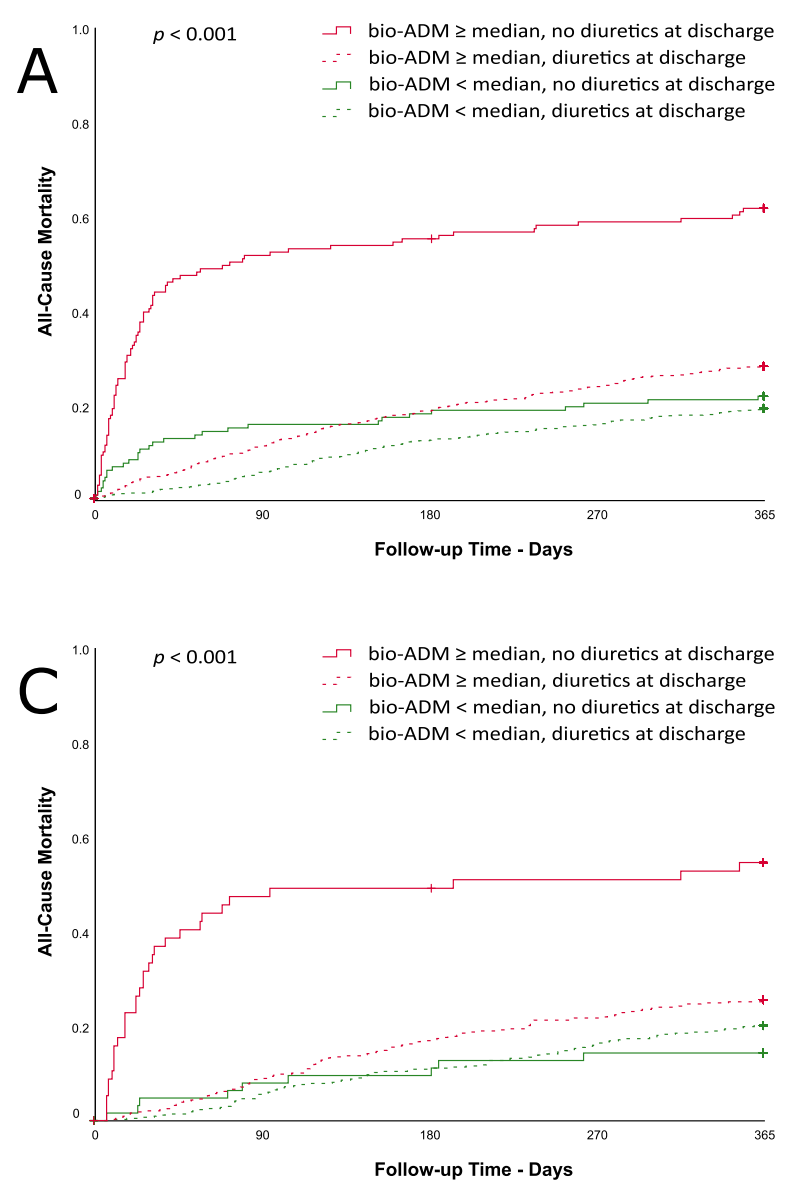

Fig. 1 Mortality stratified according to bio-ADM and MR-pro-ADM concentration and the use of diuretics at discharge: A bio-ADM at presentation $(n=1844)$; B MR-proADM at presentation $(n=738)$; C bio-ADM at discharge $(n=997)$; D MR-proADM at discharge

when arterial hypovolemia is considered the most likely cause of the absence of diuretic therapy at discharge [18, $28,29]$. Whenever medical reasons possibly justifying temporal withdrawal of diuretics, such as severe renal dysfunction or hypotension, are no longer present at discharge and in the vulnerable transition period immediately
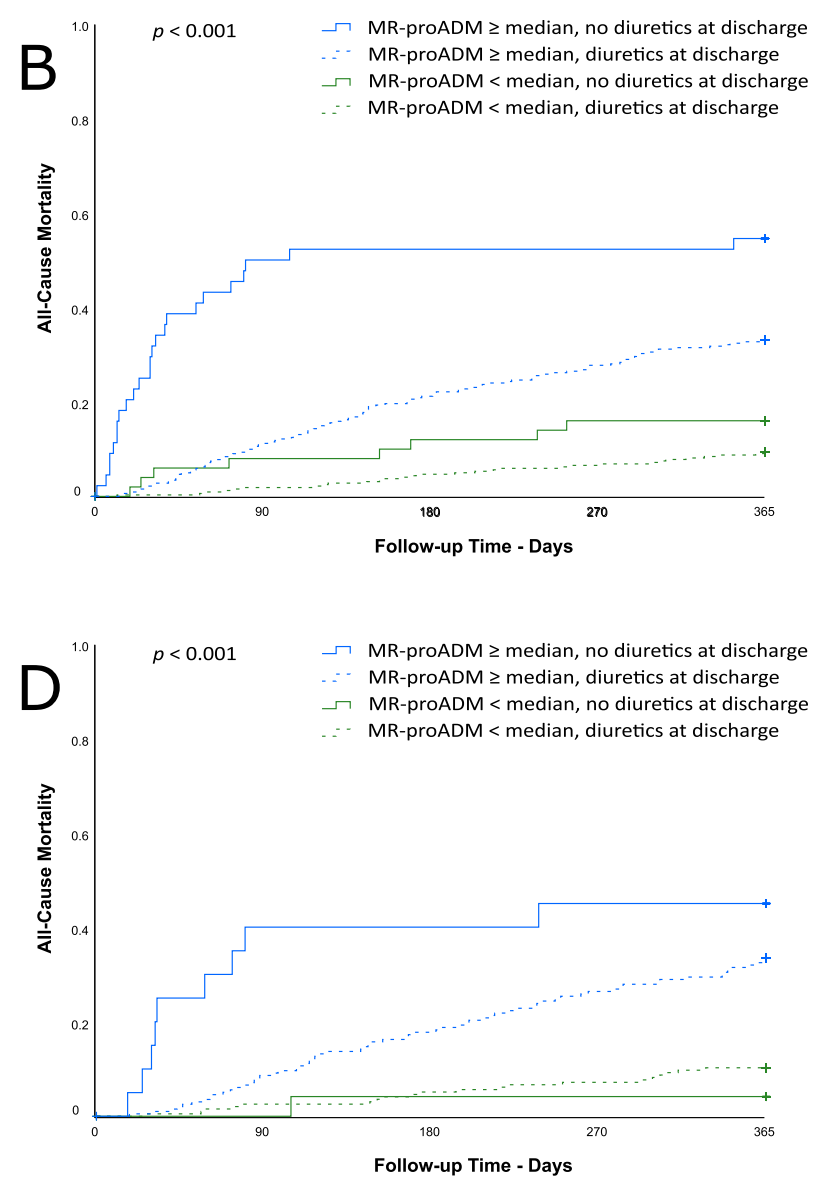

( $n=438) . A C E$ angiotensin-converting-enzyme, ARBs angiotensin receptor blocker, bio- $A D M$ bioactive adrenomedullin, $M R$-proADM midregional proadrenomedullin

after discharge, reinstitution of diuretics seems to have major importance particularly in patients identified to be at very high risk of death by elevated bio-ADM concentrations $[17,30]$. The value of bio-ADM in this setting is reinforced by the challenge to document euvolemia in hospitalised AHF patients [12]. As therapy for AHF at 
Fig. 2 Time-dependent ROC curves describing the prognostic performance of bio-ADM and MR-proADM to predict death during 365-day follow-up $(n=764)$. AUC of ROC curve: area under the time-dependent receiver-operating characteristic curve. bio-ADM bioactive adrenomedullin, $M R$-proADM midregional proadrenomedullin

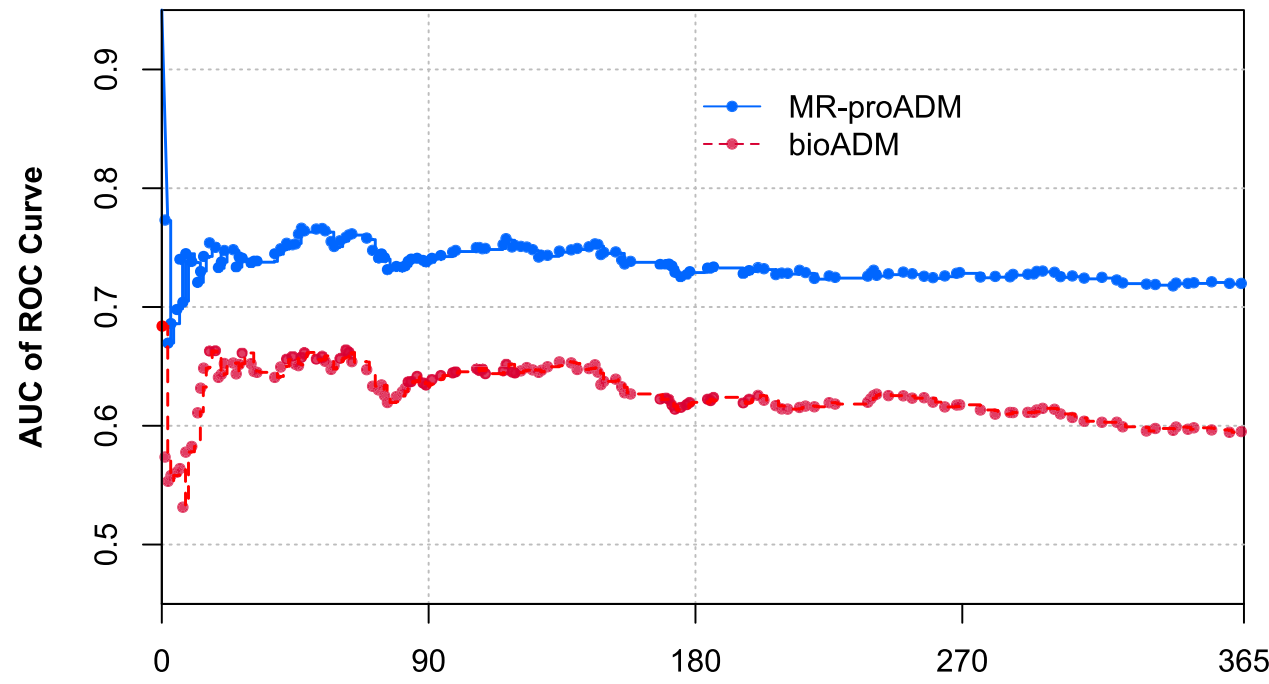

Follow-Up Time - Days discharge often remains unchanged for several weeks and even months after discharge [1, 31], over-restrictive use of diuretics at this critical time point seems to have detrimental consequences for patients.

The statistically significant interaction of bio-ADM and MR-proADM with benefits from diuretics at discharge is well in line with recent evidence from a large international study characterising bio-ADM concentrations as a quantitative marker of residual congestion in heart failure [12, 30]. Interestingly, the link between bio-ADM and residual tissue congestion and/or benefit from diuretics after discharge seems to be stronger compared to that for NT-proBNP, which mainly reflects increased left ventricular filling pressures and intravascular volume overload [12]. This might be related to the important role of the adrenomedullin system in maintaining endothelial barrier function through regulation of the cortical actin formation in endothelial cells $[15,32,33]$. In vitro analysis showed that dysfunction of the adrenomedullin system leads to enhanced vascular permeability, and subsequently, pronounced oedema [33]. Accordingly, the unique ability of adrenomedullin to reflect this specific pathophysiological pathway in AHF could explain its incremental value to the established OPTIMIZE-HF clinical risk-prediction score [21].

The reason why these two biochemical approaches providing insight into the adrenomedullin system showed different results in the prediction of death and in the identification of patients who would most benefit from diuretic therapy at discharge remains largely unknown. The biological active bio-ADM was superior in the latter aspect as not only discharge, but already admission concentrations allowed the identification of patients with particular benefit from longterm diuretic therapy. In contrast, MR-proADM was superior in predicting death which might be explained by the stronger correlation of MR-proADM with age and creatinine values compared to bio-ADM.

The current study has several limitations. First, our findings were based on a large number of patients prospectively enrolled and hospitalised in three European countries. Further research is warranted to validate our findings in other European and non-European populations. Second, our results cannot be extrapolated to patients with terminal renal failure undergoing long-term haemodialysis, because they were excluded. Third, although patients were enrolled prospectively at all three sites, some variables including biomarker measurements at discharge were not available for patients enrolled in Paris. Fourth, data on dose of diuretics as well as doses of guidelines directed medical treatment for chronic heart failure was not available. Fifth, we could not assess the possible interaction between bio-ADM and MR-proADM concentrations and sacubitril/valsartan, as this drug was not yet in clinical use at patients' enrolment. Sixth, despite indepth review of the individual patient's characteristics including severe renal dysfunction and/or hypotension, the final reasons why AHF patients were not prescribed with diuretics at discharge cannot be established. Seventh, statistically significant interaction does not prove causality. Therefore, only a randomised controlled intervention study can prove improved outcomes with the use of diuretics post-discharge in AHF patients guided by adrenomedullin system activity.

In conclusion, quantification of adrenomedullin system activity seems to enable accurate risk-prediction in AHF patients and identify inadequate decongestion prior to discharge, thereby has the potential to facilitate personalised post-discharge diuretic treatment.

Supplementary Information The online version contains supplementary material available at https://doi.org/10.1007/s00392-021-01909-9. 
Acknowledgements We thank the patients who participated in the study, the staff of the emergency departments and the laboratory technicians.

Funding Open Access funding provided by Universität Basel (Universitätsbibliothek Basel). This work was supported by research grants from the European Union, the Swiss National Science Foundation, the Swiss Heart Foundation, the Cardiovascular Research Foundation Basel, the University of Basel, the University Hospital Basel, Critical Diagnostics, Abbott, Alere, BRAHMS, Roche, and Singulex. The sponsors had no role in the design and conduct of the study; collection, management, analysis, and interpretation of the data; and preparation, review, or approval of the manuscript.

Data availability The data that support the findings of this study are available from the corresponding author upon reasonable request.

\section{Code availability Not applicable.}

\section{Declarations}

Conflict of interest Christian Mueller, as principal investigator, was supported by grants from the Swiss National Science Foundation (PP00B-102853), the Swiss Heart Foundation, the University of Basel, Abbott, ALERE, BRAHMS, and Pronota. In addition, Christian Mueller received lecture fees from Abbott, ALERE, BRAHMS, Novartis, and Roche. Nikola Kozhuharov was supported by grants from the Swiss National Science Foundation (P400PM-194477), Gottfried und Julia Bangerter-Rhyner-Stiftung, and the European Society of Cardiology. Iain Squire reports grants and personal fees from NOVARTIS, grants and personal fees from VIFOR, grants from BOEHRINGER INGELHEIM, grants from ASTRA ZENECA, grants from MERCK, outside the submitted work. Andreas Bergmann is the director of Sphingotec GmbH which is the manufacturer of Sphingotest bio-ADM diagnostic test. Joachim Struck and Oliver Hartmann are employed by Sphingotec GmbH. Christian Puelacher reports grants from Roche Diagnostics, outside the submitted work. Dr. Mebazaa reports personal fees from Orion, grants and personal fees from Roche, personal fees from Servier, personal fees from Otsuka, personal fees from Philips, grants and personal fees from Adrenomed, personal fees from Neuro Tronik, grants and personal fees from 4TEEN4, personal fees from Sanofi, outside the submitted work; and Alexandre Mebazaa owns shares of S-Form Pharma. Dr. Breidthardt has received research grants from the Swiss National Science Foundation (PASMP3-134362), the University Hospital Basel, the Department of Internal Medicine, University Hospital Basel, Abbott, and Roche as well as speaker honoraria from Roche. All the other authors declare that they have no conflict of interest with this study.

Ethical approval This study complied with the Declaration of Helsinki and ethics approval was granted from the respective research ethics committees.

Consent to participate All the patients provided written informed consent.

Consent for publication All the authors have read and approved the manuscript.

Open Access This article is licensed under a Creative Commons Attribution 4.0 International License, which permits use, sharing, adaptation, distribution and reproduction in any medium or format, as long as you give appropriate credit to the original author(s) and the source, provide a link to the Creative Commons licence, and indicate if changes were made. The images or other third party material in this article are included in the article's Creative Commons licence, unless indicated otherwise in a credit line to the material. If material is not included in the article's Creative Commons licence and your intended use is not permitted by statutory regulation or exceeds the permitted use, you will need to obtain permission directly from the copyright holder. To view a copy of this licence, visit http://creativecommons.org/licenses/by/4.0/.

\section{References}

1. Miró Ò, Peacock FW, McMurray JJ et al (2017) European Society of Cardiology - acute cardiovascular care association position paper on safe discharge of acute heart failure patients from the emergency department. Eur Hear J Acute Cardiovasc Care 6:311-320. https://doi.org/10.1177/2048872616633853

2. Storrow AB, Jenkins CA, Self WH et al (2014) The burden of acute heart failure on US emergency departments. JACC Heart Fail 2:269-277. https://doi.org/10.1016/j.jchf.2014.01.006

3. Mueller C, Christ M, Cowie M et al (2017) European Society of Cardiology - acute cardiovascular care association position paper on acute heart failure: a call for interdisciplinary care. Eur Heart J Acute Cardiovasc Care 6:81-86. https://doi.org/10. $1177 / 2048872615593279$

4. Maisel AS, Krishnaswamy P, Nowak RM et al (2002) Rapid measurement of B-type natriuretic peptide in the emergency diagnosis of heart failure. N Engl J Med 347:161-167. https:// doi.org/10.1056/NEJMoa020233

5. Mueller C, Scholer A, Laule-Kilian K et al (2004) Use of B-type natriuretic peptide in the evaluation and management of acute dyspnea. $\mathrm{N}$ Engl J Med 350:647-654. https://doi.org/10.1056/NEJMoa031681

6. Ponikowski P, Voors AA, Anker SD et al (2016) 2016 ESC guidelines for the diagnosis and treatment of acute and chronic heart failure. Eur Heart J 37:2129-2200. https://doi.org/10. 1093/eurheartj/ehw128

7. Rutten JHW, Steyerberg EW, Boomsma F et al (2008) N-terminal pro-brain natriuretic peptide testing in the emergency department: beneficial effects on hospitalization, costs, and outcome. Am Heart J 156:71-77. https://doi.org/10.1016/j.ahj. 2008.02.021

8. Jougasaki M, Burnett JC (2000) Adrenomedullin: potential in physiology and pathophysiology. Life Sci 66:855-872

9. Maisel A, Mueller C, Nowak RM et al (2011) Midregion prohormone adrenomedullin and prognosis in patients presenting with acute dyspnea: results from the BACH (biomarkers in acute heart failure) trial. J Am Coll Cardiol 58:1057-1067. https://doi.org/10. 1016/j.jacc.2011.06.006

10. Pousset F, Masson F, Chavirovskaia O et al (2000) Plasma adrenomedullin, a new independent predictor of prognosis in patients with chronic heart failure. Eur Heart J 21:1009-1014. https://doi. org/10.1053/euhj.1999.1904

11. Nishikimi T, Saito Y, Kitamura K et al (1995) Increased plasma levels of adrenomedullin in patients with heart failure. J Am Coll Cardiol 26:1424-1431. https://doi.org/10.1016/0735-1097(95)00338-X

12. ter Maaten JM, Kremer D, Demissei BG et al (2019) Bio-adrenomedullin as a marker of congestion in patients with new-onset and worsening heart failure. Eur J Heart Fail 21:732-743. https:// doi.org/10.1002/ejhf.1437

13. Pandhi P, ter Maaten JM, Emmens JE et al (2019) Clinical value of pre-discharge bio-adrenomedullin as a marker of residual congestion and high risk of heart failure hospital readmission. Eur J Heart Fail 22:683-691. https://doi.org/10.1002/ejhf.1693 
14. Hirano S, Imamura T, Matsuo T et al (2000) Differential responses of circulating and tissue adrenomedullin and gene expression to volume overload. J Card Fail 6:120-129. https://doi.org/10.1054/jcaf.2000.7277

15. Voors AA, Kremer D, Geven C et al (2019) Adrenomedullin in heart failure: pathophysiology and therapeutic application. Eur J Heart Fail 21:163-171. https://doi.org/10.1002/ejhf.1366

16. Ellison DH, Felker GM (2017) Diuretic treatment in heart failure. $\mathrm{N}$ Engl J Med 377:1964-1975. https://doi.org/10.1056/nejmra1703100

17. Self WH, Storrow AB, Hartmann O et al (2016) Plasma bioactive adrenomedullin as a prognostic biomarker in acute heart failure. Am J Emerg Med 34:257-262. https://doi.org/10.1016/j.ajem. 2015.10.033

18. Breidthardt T, Weidmann ZM, Twerenbold R et al (2017) Impact of haemoconcentration during acute heart failure therapy on mortality and its relationship with worsening renal function. Eur J Heart Fail 19:226-236. https://doi.org/10.1002/ejhf.667

19. Weber J, Sachse J, Bergmann S et al (2017) Sandwich immunoassay for bioactive plasma adrenomedullin. J Appl Lab Med 2:222-233. https://doi.org/10.1373/jalm.2017.023655

20. Caruhel P, Mazier C, Kunde J et al (2009) Homogeneous timeresolved fluoroimmunoassay for the measurement of midregional proadrenomedullin in plasma on the fully automated system B.R.A.H.M.S KRYPTOR®. Clin Biochem 42:725-728. https:// doi.org/10.1016/j.clinbiochem.2009.01.002

21. O'Connor CM, Abraham WT, Albert NM et al (2008) Predictors of mortality after discharge in patients hospitalized with heart failure: an analysis from the organized program to initiate lifesaving treatment in hospitalized patients with heart failure (OPTIMIZE-HF). Am Heart J 156:662-673. https://doi.org/10.1016/j.ahj.2008.04.030

22. Voors AA, Ouwerkerk W, Zannad F et al (2017) Development and validation of multivariable models to predict mortality and hospitalization in patients with heart failure. Eur J Heart Fail 19:627-634. https://doi.org/10.1002/ejhf.785

23. Kamarudin AN, Cox T, Kolamunnage-Dona R (2017) Timedependent ROC curve analysis in medical research: current methods and applications. BMC Med Res Methodol 17:53. https://doi. org/10.1186/s12874-017-0332-6

24. Breidthardt T, Balmelli C, Twerenbold R et al (2013) Heart failure therapy-induced early ST2 changes may offer long-term therapy guidance. J Card Fail 19:821-828. https://doi.org/10.1016/j.cardfail.2013.11.003
25. Morbach C, Marx A, Kaspar M et al (2017) Prognostic potential of midregional pro-adrenomedullin following decompensation for systolic heart failure: comparison with cardiac natriuretic peptides. Eur J Heart Fail 19:1166-1175. https://doi.org/10.1002/ejhf.859

26. Molvin J, Jujic A, Navarin S et al (2019) Bioactive adrenomedullin, proenkephalin A and clinical outcomes in an acute heart failure setting. Open Heart 6:e001048. https://doi.org/10.1136/openhrt-2019-001048

27. Stienen S, Salah K, Dickhoff C et al (2015) N-terminal pro-Btype natriuretic peptide (NT-proBNP) measurements until a $30 \%$ reduction is attained during acute decompensated heart failure admissions and comparison with discharge NT-proBNP levels: implications for in-hospital guidance of treatment. J Card Fail 21:930-934. https://doi.org/10.1016/j.cardfail.2015.07.011

28. Breidthardt T, Socrates T, Noveanu M et al (2011) Effect and clinical prediction of worsening renal function in acute decompensated heart failure. Am J Cardiol 107:730-735. https://doi.org/ 10.1016/j.amjcard.2010.10.056

29. Mullens W, Damman K, Harjola V-P et al (2019) The use of diuretics in heart failure with congestion - a position statement from the Heart Failure Association of the European Society of Cardiology. Eur J Heart Fail 21:137-155. https://doi.org/10.1002/ejhf.1369

30. Kremer D, ter Maaten JM, Voors AA (2018) Bio-adrenomedullin as a potential quick, reliable, and objective marker of congestion in heart failure. Eur J Heart Fail 20:1363-1365. https://doi.org/10.1002/ejhf.1245

31. Harjola V-P, Parissis J, Brunner-La Rocca H-P et al (2018) Comprehensive in-hospital monitoring in acute heart failure: applications for clinical practice and future directions for research. A statement from the Acute Heart Failure Committee of the Heart Failure Association (HFA) of the European Society of Cardio. Eur J Heart Fail 20:1081-1099. https://doi.org/10.1002/ejhf.1204

32. Temmesfeld-Wollbrück B, Hocke AC, Suttorp N, Hippenstiel S (2007) Adrenomedullin and endothelial barrier function. Thromb Haemost 98:944-951

33. Koyama T, Ochoa-Callejero L, Sakurai T et al (2013) Vascular endothelial adrenomedullin-RAMP2 system is essential for vascular integrity and organ homeostasis. Circulation 127:842-853. https://doi.org/10.1161/CIRCULATIONAHA.112.000756

\title{
Authors and Affiliations
}

\author{
Nikola Kozhuharov $v^{1,2,3}$. Leong $\mathrm{Ng}^{4,5}$. Desiree Wussler ${ }^{1,2,6} \cdot$ Ivo Strebel $^{1,2}$. Zaid Sabti ${ }^{1,2}$. Oliver Hartmann ${ }^{7}$. \\ Mohamed Eltayeb ${ }^{4,5}$. lain Squire ${ }^{4,5}$. Albina Nowak ${ }^{1,2,8} \cdot$ Max Rieger $^{1,2} \cdot$ Jasmin Martin $^{1,2,6}$. Eleni Michou ${ }^{1,2}$. \\ Sabrina Stefanelli ${ }^{1,2}$. Christian Puelacher ${ }^{1,2,6} \cdot$ Samyut Shrestha ${ }^{1,2} \cdot$ Maria Belkin $^{1,2} \cdot$ Tobias Zimmermann $^{1,2,6}$. \\ Pedro Lopez-Ayala ${ }^{1,2}$. Joachim Struck ${ }^{7}$. Andreas Bergmann ${ }^{7}$. Alexandre Mebazaa ${ }^{9}$. Alice Blet ${ }^{9}$. \\ Danielle Menosi Gualandro ${ }^{1,2} \cdot$ Tobias Breidthardt $^{1,2,6} \cdot$ Christian Mueller $^{1,2}($ )
}

1 Department of Cardiology and Cardiovascular Research Institute Basel (CRIB), University Hospital Basel and University of Basel, Petersgraben 4, CH-4031 Basel, Switzerland

2 GREAT Network, Rome, Italy

3 Institute of Cardiovascular Medicine and Science, Liverpool Heart and Chest Hospital, Liverpool, UK

4 Department of Cardiovascular Sciences, University of Leicester, Leicester, UK

5 NIHR Leicester Cardiovascular Biomedical Research Unit, Glenfield Hospital, Leicester, UK
6 Department of Internal Medicine, University Hospital Basel, University of Basel, Basel, Switzerland

7 Sphingotec GmbH, Hennigsdorf, Germany

8 Department of Endocrinology and Clinical Nutrition, University Hospital Zürich, University of Zürich, Zürich, Switzerland

9 University Paris Diderot, APHP Hôpitaux Universitaires Saint Louis Lariboisière, Inserm 942, Paris, France 